\title{
Creation of the precision magnetic spectrometer SCAN-3
}

\author{
S.V. Afanasiev ${ }^{1, \star}$, Yu.S. Anisimov ${ }^{1}$, A.A. Baldin ${ }^{1,2}$, A.I. Berlev ${ }^{1,2}$, D.K. Dryablov ${ }^{1, \star}$, \\ B.V. Dubinchik ${ }^{1}$, A.F. Elishev ${ }^{1}$, O.V. Fateev, ${ }^{1}$, Z.A. Igamkulov ${ }^{1}$, Yu.F. Krechetov ${ }^{1}$, I.V. Kudashkin ${ }^{1,2}$, \\ S.N. Kuznechov ${ }^{1}$, A.I. Malakhov ${ }^{1}$, V.A. Smirnov ${ }^{1}$, S.S. Shimansky ${ }^{1}$, J. Kliman ${ }^{3}$, V. Matousek ${ }^{3}$, \\ S. Gmutsa ${ }^{3}$, I. Turzo ${ }^{3}$, I. Cruceru ${ }^{4}$, M. Cruceru ${ }^{4}$, F. Constantin ${ }^{4}$, G. Niolescu ${ }^{4}$, \\ L. Ciolacu ${ }^{4}$, M. Paraipan $^{5}$, S. Vokál, ${ }^{6}$, J. Vrláková6, V.A. Baskov, ${ }^{7}$, A.I. Lebedev ${ }^{7}$, \\ A.I. L'vov', L.N. Pavlyuchenko ${ }^{7}$, V.V. Polyansky" ${ }^{7}$, E.V. Rzhanov ${ }^{7}$, S.S. Sidorin ${ }^{7}$, G.A. Sokol ${ }^{7}$, \\ I.V. Glavanakov ${ }^{8}$, A.N. Tabachenko ${ }^{8}$, D.M. Jomurodov ${ }^{9}$, R.N. Bekmirzaev ${ }^{9}$, R.M. Ibadov ${ }^{10}$, and \\ M.U. Sultanov ${ }^{10}$ \\ 1 Joint Institute for Nuclear Research, Dubna, Russia \\ ${ }^{2}$ Institute advanced studies «OMEGA», Dubna, Russia \\ ${ }^{3}$ Institute of Physics, Slovak Academy of Sciences, Slovak republic \\ ${ }^{4}$ Horia Hulubei National Institute of R\&D for Physics and Nuclear engineering, IFIN-HH Bucharest, Romania \\ ${ }^{5}$ Institute for Space Science, Bucharest-Magurele, Romania \\ ${ }^{6}$ Faculty of Science, University of P.J. Šafárik, Košice, Slovak republic \\ ${ }^{7}$ Lebedev Physical Institute, Moscow, Russia \\ ${ }^{8}$ Tomsk Polytechnic University, Tomsk, Russia \\ ${ }^{9}$ Jizzakh State Pedagogical Institute, Uzbekistan \\ ${ }^{10}$ Samarkand State University, Uzbekistan
}

\begin{abstract}
The new JINR project [1] is aimed at studies of highly excited nuclear matter created in nuclei by a high-energy deuteron beam. The matter is studied through observation of its particular decay products - pairs of energetic particles with a wide opening angle, close to $180^{\circ}$. The new precision hybrid magnetic spectrometer SCAN-3 is to be built for detecting charged $\left(\pi^{ \pm}, K^{ \pm}, \mathrm{p}\right)$ and neutral (n) particles produced at the JINR Nuclotron internal target in dA collisions. One of the main and complex tasks is a study of low-energy $\eta \mathrm{A}$ interaction and a search for $\eta$-bound states ( $\eta$-mesic nuclei). Basic elements of the spectrometer and its characteristics are discussed in the article.
\end{abstract}

\section{Introduction}

Among the lightest pseudoscalar mesons the eta (and eta-prime) are very interesting objects because their properties are strongly influenced by a flavour SU(3) singlet component, presumably made of gluons, as the axial U(1) anomaly suggests. Masses and decay widths of these mesons, as well as their interactions with nucleons and nuclei, crucially depend on the singlet component that is affected by the nuclear environment. In this way studies of $\eta \mathrm{N}$ and $\eta \mathrm{A}$ interactions become related with very fundamental aspects of nuclear physics and QCD [2].

\footnotetext{
${ }^{\star}$ e-mail: afanasev@lhe.jinr.ru

$\star \star$ e-mail: dryablov@1he.jinr.ru
} 
One of the main aims of the experiment SCAN-3 at the internal deuteron beam of the JINR Nuclotron is directed to studies of the $\eta$ A system. More specifically, we will study quasi-bound states of the $\eta$ in nuclei first predicted in theoretical works by Haider an Liu [3], who discovered that $\eta \mathrm{N}$ and $\eta \mathrm{A}$ interaction is attractive at low energies.

The experiment SCAN-3 is aimed at investigations of dA collisions and observation of the fast decay products of the produced bound $\eta$ in nuclei, $\pi \mathrm{N}$ and $\mathrm{NN}$ pairs, caused by the internal annihilationat-rest reactions $\eta \mathrm{N} \rightarrow \pi \mathrm{N}$ and $\eta \mathrm{NN} \rightarrow \mathrm{NN}$. Information on the binding of $\eta$ and eventually the lowenergy $\eta \mathrm{A}$ interaction can be inferred from precise measurements of the energy spectra of the $\eta \mathrm{N}$ and NN pairs. Principles of this approach are explained in more detail [4].

In 2006-2010 a set of experimental data at two arms time-of-flight setup SCAN-2 and the internal d-beam of Nuclotron was obtained. The JINR collaboration performed the search for back-to-back $\pi \mathrm{p}$ pairs related to the $\eta$-mesic bound states in $d+{ }^{12} C \rightarrow \pi+p+X$ process [5]. An observation of the $\pi \mathrm{p}$ back-to-back correlation as well as the resonance like structure below $\eta$ production threshold could be associated with the two-body $\mathrm{N}^{*}$ resonance decay related with formation of an $\eta$-mesic nucleus. The new project SCAN-3 provides a significant increase of the energy resolution of the setup by including a magnetic spectrometer in the experimental installation and by increasing the time-of-flight base.

\section{Requirements of the experiment}

\subsection{What we are looking for?}

The interaction of the $\eta$-meson with a nucleon near threshold is mainly determined by the $\mathrm{S}_{11}, \mathrm{~J}^{P}=\frac{1}{2}^{-}$ resonance $\mathrm{N}^{*}(1535)$, which is just $49 \mathrm{MeV}$ above the $\eta \mathrm{N}$ threshold $(1486 \mathrm{MeV})$ and has a width $\Gamma=150 \mathrm{MeV}$, thus covering the whole low energy region of the $\eta \mathrm{N}$ interaction. The $\mathrm{S}_{11}$ resonance also decays to $\pi \mathrm{N}, \gamma \mathrm{N}$ and $\pi \pi \mathrm{N}$ channels. It is important to recognize that if we register $\pi \mathrm{N}$ pair with approximately equal but opposite momentum components, even with some suitable total energy $\mathrm{E}_{\pi}+$ $\mathrm{E}_{p} \approx \mathrm{m}_{\eta}+\mathrm{m}_{N}=1486 \mathrm{MeV}$, it does not necessarily mean that we have registered the decay products of the $\eta$-mesic nucleus.

Generally [4], observation of a relatively narrow resonance peak in the spectrum of $E_{\eta}$ in the subthreshold region $\mathrm{E}_{\eta}<\mathrm{m}_{\eta}$ is mandatory for claiming an observation of $\eta$-mesic nuclei at all.

\subsection{Requirements to precisions}

Theoretical predictions for the binding energy and width of the $\eta$-nuclear levels are highly dependent on the assumed $\eta$-nuclear potential and nucleus size. Apparently, future experiments should assume that the peak width will be about $10 \mathrm{MeV}$, and therefore they should provide accurate measurements of particle energies not worse than $\sim 3-5 \mathrm{MeV}$, so that the accuracy of the total energy of the pair will be at least 5-7 MeV.

In our experiment, the requirement of accuracy of energy determination is weakened (approximately in $\sim 1.5$ times). It is caused by effects of a broadening of observable peak in a total energy spectrum of the correlated pair, caused by energy dispersion of intra-nuclear nucleons on which an annihilation of bounded meson is realized. This dispersion additionally increases observable width of peak by $\sim 20 \mathrm{MeV}$. Thus the requirement to accuracy at level of 5-7 MeV is superfluous. That reduces the accuracy to the level of $10 \mathrm{MeV}$.

If we consider the process $\eta+\mathrm{N}_{i} \rightarrow \pi+\mathrm{N}$ with initial particles at rest, the kinetic energy and momentum of the secondary particles must be equal to (if we ignore the effect of binding and Fermi motion): $\mathrm{E}_{\pi}=313 \mathrm{MeV}, \mathrm{E}_{N}=94 \mathrm{MeV}, \mathrm{p}_{\pi}=\mathrm{p}_{N}=431 \mathrm{MeV} / \mathrm{c}$. Accordingly, experiment performance demands measurement precisions of kinetic energies at level of $1 \%$ for such of pions and $3 \%$ for such 
of nucleons. If we consider the process $\eta+\mathrm{N}_{i}+\mathrm{N}_{j} \rightarrow \mathrm{N}_{1}+\mathrm{N}_{2}$, the kinetic energy and momentum are about $\mathrm{E}_{1}=\mathrm{E}_{2}=273 \mathrm{MeV}, \mathrm{p}_{1}=\mathrm{p}_{2}=767 \mathrm{MeV} / \mathrm{c}$. Accordingly, experiment performance at the declared level demands accuracy in measuring of kinetic energies of such nucleons at level of $1 \%$.

\section{Layout of the setup}

We propose to extend a setup used at the Nuclotron in our previous studies in order to achieve the energy resolution for both charged and neutral components of the correlated $\pi \mathrm{N}$ and NN pairs of $\sigma \approx$ $5 \mathrm{MeV}$. To this aim we plan to construct a magnetic spectrometer ( $\mathrm{M}$ arm) and two sectioned neutron detectors with a TOF base up to $6 \mathrm{~m}$ (P and $\mathrm{K}$ arms), see figure 1. The TOF arm (P-arm) is supposed to be used primarily for registration of protons and neutrons with the desired characteristics. The TOF technique for protons is well-proven in the previous experimental setup SCAN-2. Magnetic arm (M-arm) will be used for registration of pions. Technical parameters of the detectors, including their space and time resolution, have been specified using GEANT simulations [6]. Data acquisition system is to be built using VME modules produced in JINR.

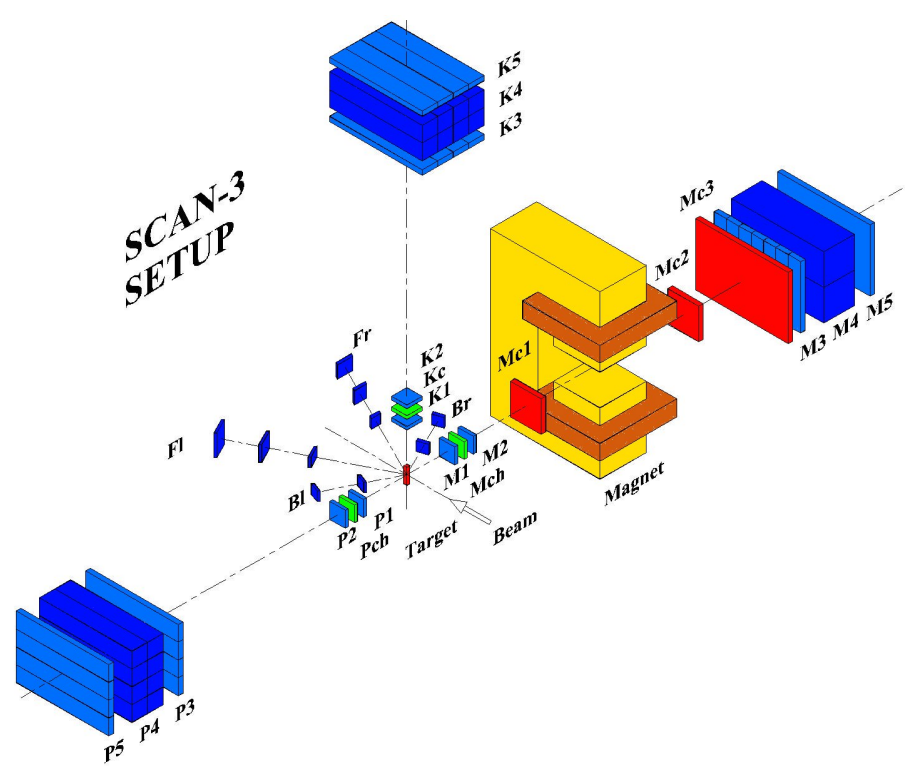

Figure 1. Layout of the SCAN-3 setup. Fr, Fl, Br, B1 - scintillator monitor counters. P1-P3, K1-K3, M1-M3 trigger time-of-flight scintillator detectors. Pch, Kch, Mch - threshold Cherenkov detectors. Mc1, Mc2 - drift chambers. Mc3 - multiwire proportional chamber. M4 -scintillator total absorption detector. P4, K4 - two sets of scintillator neutron counters. P5, K5, M5 - veto detectors.

\section{Magnetic system}

At the moment, multiwire proportional chamber (Mc3) is ready for use, two biplanar drift chambers $(\mathrm{Mc} 1$ and $\mathrm{Mc} 2)$ are simulated, the magnet $\left(\mathrm{B}_{\max }=7 \mathrm{kG}\right)$ is installed in the area of the Nuclotron internal target. 

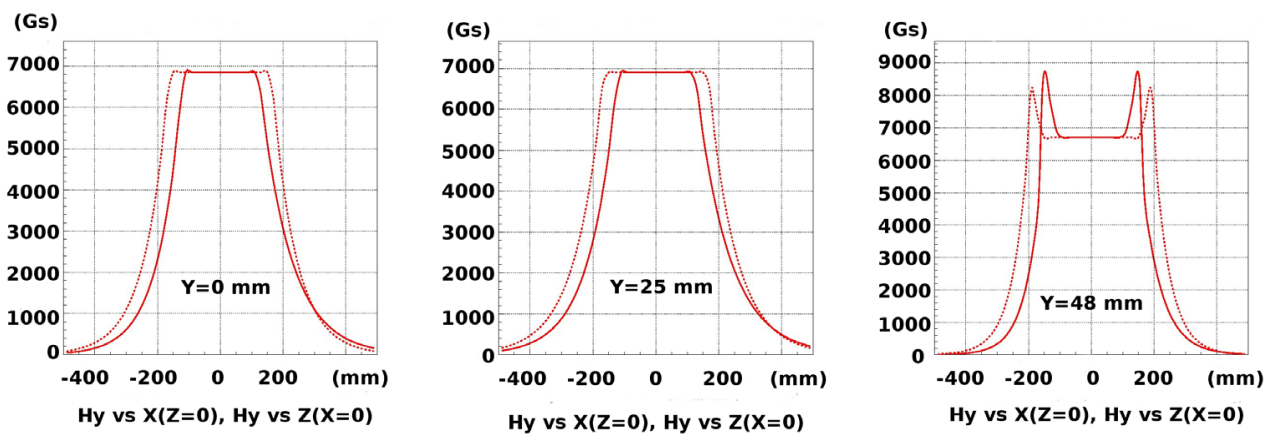

Figure 2. The magnetic field of the SP-46. The dependence of the vertical component of the magnetic field Hy (in Gs) on the coordinates $\mathrm{X}$ (axis is perpendicular to the average momentum of the analyzed particles) and $\mathrm{Z}$ (axis is parallel to the average momentum of the analyzed particles) in the planes: $Y=0 \mathrm{~mm}$ (left figure), $\mathrm{Y}=25$ $\mathrm{mm}$ (middle figure), $\mathrm{Y}=48 \mathrm{~mm}$ (right figure).

The use of the magnet SP-46 is a good offer for a magnetic spectrometer. Parameters of the magnet are: the transverse gap - 100 (vertical direction) x 300 (horizontal direction) $\mathrm{mm}^{2}$, magnetic field $7 \mathrm{kGs}$ at the current of $20 \mathrm{~A}$, the length of the magnetic track - 420mm. The vertical gap of SP-46 can be very easily increased from $100 \mathrm{~mm}$ to $170 \mathrm{~mm}$ by removing the $35 \mathrm{~mm}$ flat parts of the magnet (the field will drop to $\sim 5 \mathrm{kGs}$ ). The magnetic field was measured in the case of series-connected coils (total resistance of $2.5 \mathrm{Ohm}$ ) and current $\mathrm{I}=20 \mathrm{~A}$. Some results of magnetic field measurements are shown in the figure 2.

We have analyzed the accuracy of determining of the deflection angle of $\pi$ and $p$ in the magnetic field depending on the properties of the detectors, the geometry and materials. In this case we have not considered the boundary perturbations of the magnetic field. It is necessary for further simulations. Data analysis for pions has shown that the accuracy of the angular resolution could be better, then $1.4 \%$ (it corresponds to the pion kinetic energy of $4.4 \mathrm{MeV}$ ). Data analysis for protons has shown that the accuracy of the angular resolution could be better than $2.4 \%$ (it corresponds to the proton kinetic energy of $6.5 \mathrm{MeV}$ ).

The figure 3 shows the normalized momentum distributions of protons and pions in the M-arm. Reducing the particle detection efficiency at low momenta is justified by the fact that particles do not reach the detectors $\mathrm{P} 3$ or $\mathrm{K} 3$, which are included in the trigger.

\section{Neutron detectors}

P1-P3, M1-M3 time-of-flight scintillator detectors need to be calibrated. Prototype of a neutron detector is tested. The figure 4 shows geometry of the neutron detector.

The energy of the neutron is determined by time of flight on the basis of up to $6 \mathrm{~m}$. The neutron detector will consist of several elements. Each element is an assembly of four long plastic scintillator with a thickness of $3 \mathrm{~cm}$. The ends of each element are connected to a fast PMT with a photocathode diameter of about $100 \mathrm{~mm}$. The ends of each of the four plates are further connected to the photomultiplier with a diameter of about $2 \mathrm{~cm}$ to identify the plate number. Simulation showed that the 

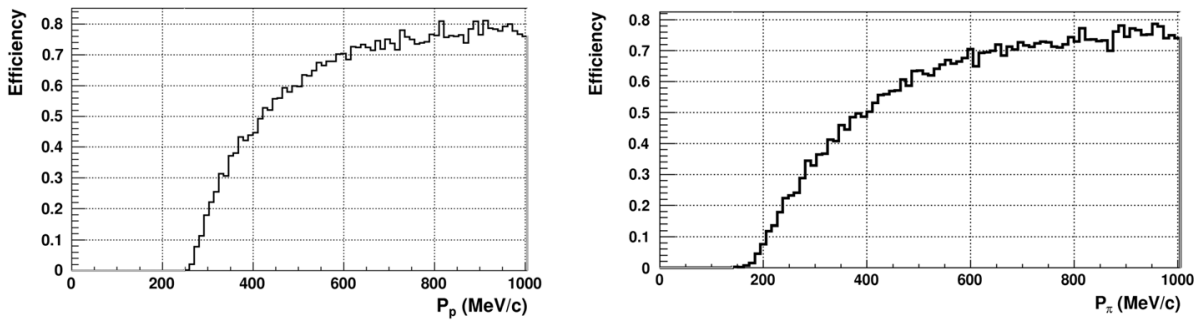

Figure 3. Momentum efficiency of protons (left) and pions (right) in the M-arm of the SCAN3.
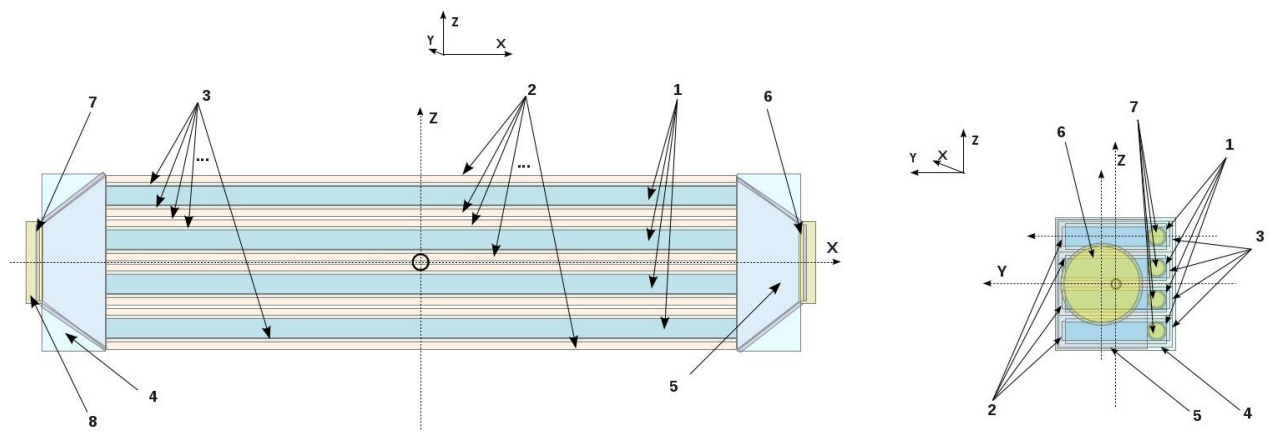

Figure 4. Geometry of the neutron detector: 1 - plates of organic scintillator (NE110 type, optics), 2 - air gap, 3 - wrap material for plates (synthetic paper tyvek, optics), 4 - air volume of the fiber (optics), 5 - reflecting wall of the optical fiber (aluminized Mylar $0.3 \mathrm{~mm}$, optics), 6 - photocathode of PMT (XP2041 type), 7 - photocathode of PMT (PMT-85 type).

required energy resolution of neutron (about $5 \mathrm{MeV}$ ) is achieved with a time resolution of the neutron detector of 200 ps. Experimental study showed that the photomultiplier XP2020 is the most suitable, it provides a time resolution of about $250-280 \mathrm{ps}$.

To improve time resolution, it was proposed to use a PMT from the system identification plate. The researchers have studied the time characteristics of the FEU-85, FEU-87 with different voltage dividers. FEU-87 showed approximately $20 \%$ better time resolution than PMT-85, however, the light collection of PMT-85 about $30 \%$ higher.

It was found that the simultaneous use of the information from XP2020 and PMT-87 (or PMT-85) allows us to reach the required level of temporary and, therefore, the energy resolution of neutron detector. Testing of the detector continues.

\section{Conclusions}

The first main aims are:

- building of a magnetic spectrometer for detection of charged components of the correlated pairs with 
the energy resolution of 4-5 MeV;

- building of a precision time-of-flight neutron detector with a similar energy resolution;

SCAN-3 will allow to carry out the following studies:

- studies of the $S_{11}(1535)$ and $\Delta(1232)$ resonances in the nuclear matter;

- a search for $\eta$-nuclei and $\Delta$-nuclei in dA-collisions through narrow resonance peaks in the total energy spectra of the correlated pairs;

- determination of binding energies and widths of $\eta$ in nuclei;

- measurements of the cross section $\sigma\left({ }_{\eta} \mathrm{A}\right)$ of $\eta$-nuclei formation in dA collisions; measurements of the A-dependence of $\sigma\left({ }_{\eta} \mathrm{A}\right)$;

- measurements of relative rates of $\pi \mathrm{p}$ and $\mathrm{pN}$ events;

- measurements of the cross section of $\Delta$-nuclei formation in dA collisions;

- determination of binding energies and widths of quasi-bound states of $\Delta$ in nuclei;

- measurements of relative rates of $\pi^{+} \mathrm{p}, \pi^{-} \mathrm{p}$ and $\mathrm{pN}$ events.

\section{References}

[1] 45th meeting of the JINR Programme Advisory Committees for Particle Physics: http://indico.jinr.ru/materialDisplay.py?materialId=3\&confId $=1589$

[2] S.D. Bass and A.W. Thomas, Acta Phys. Pol. 45, 627 (2014)

[3] Q. Haider and L.C. Liu, Phys. Lett. B 172, 257 (1986)

[4] V.A.Baskov et al., e-Print: arXiv:1212.6313 [nucl-ex]

[5] S. V. Afanasiev et al., Nucl. Phys. B (Proc. Suppl.) 219-220, 255 (2011)

[6] S.V. Afanasiev et al., Int. J. Mod. Phys.: Conf. Series 39, 156009 (2015) 